\title{
Small epitaxial graphene devices for magnetosensing applications
}

\author{
V. Panchal, ${ }^{1,2}$ K. Cedergren, ${ }^{3}$ R. Yakimova, ${ }^{4}$ A. Tzalenchuk, ${ }^{1}$ S. Kubatkin, ${ }^{3}$ \\ and O. Kazakova ${ }^{1, a)}$ \\ ${ }^{1}$ NPL, Teddington, TW11 OLW, United Kingdom \\ ${ }^{2}$ RHUL, Egham, TW20 OEX, United Kingdom \\ ${ }^{3}$ Chalmers University of Technology, Göteborg, S-412 96, Sweden \\ ${ }^{4}$ Linköping University, Linköping, S-581 83, Sweden
}

(Presented 1 November 2011; received 22 September 2011; accepted 17 November 2011; published online 8 March 2012)

\begin{abstract}
Hall sensors with the width range from 0.5 to $20.0 \mu \mathrm{m}$ have been fabricated out of a monolayer graphene epitaxially grown on $\mathrm{SiC}$. The sensors have been studied at room temperature using transport and noise spectrum measurements. The minimum detectable field of a typical $10-\mu \mathrm{m}$ graphene sensor is $\approx 2.5 \mu \mathrm{T} / \sqrt{ } \mathrm{Hz}$, making them comparable with state of the art semiconductor devices of the same size and carrier concentration and superior to devices made of CVD graphene. Relatively high resistance significantly restricts performance of the smallest 500-nm devices. Carrier mobility is strongly size dependent, signifying importance of both intrinsic and extrinsic factors in the optimization of the device performance. (c) 2012 American Institute of Physics. [doi:10.1063/1.3677769]
\end{abstract}

\section{INTRODUCTION}

Small (i.e., micron and submicron sized) semiconductor Hall effect devices ${ }^{1}$ have been successfully used for magnetic field sensing in numerous industrial applications ${ }^{2-4}$ as well as fundamental research. ${ }^{5-8}$ These sensors are also widely used for detection of supera-magnetic nanobeads in environmental, biomedical and chemical applications ${ }^{9-12}$ due to their high sensitivity to a small amount of magnetic moments at room temperature and the noninvasive nature of the measurements. The essential aim is to develop a sensor with the smallest lateral dimensions possible, providing the highest spatial resolution and coupling coefficient with a nanobead. Additional improvement of the coupling can be achieved by reducing the vertical distance, $Z_{0}$, between the active channel and nanoobject. When compared to conventional $2 \mathrm{DEG}$ semiconductor devices, the active channel in graphene is at the surface and therefore $Z_{0}$ can be reduced from $\sim 100$-nm to sub-nm. While Hall sensors made of semiconductor devices are widely explored, graphene devices are relatively new for magnetosensing applications. For example, it has been recently shown that micron sized CVD graphene Hall sensors are capable of detecting magnetic fields as small as $43 \mu \mathrm{T} / \sqrt{ } / \mathrm{Hz}^{13}$

The epitaxial graphene was grown by sublimation of $\mathrm{Si}$ and subsequent graphene formation on the Si-terminated face of a nominally on-axis $4 \mathrm{H}-\mathrm{SiC}$ substrate at $2000^{\circ} \mathrm{C}$ and $1 \mathrm{bar}$ argon gas pressure. The electrodes and the Hall bars were defined by e-beam lithography in two independent steps and oxygen plasma etching was used to pattern the Hall bars. Using this method, Hall sensors with the cross width ranging from 0.5 to $20.0 \mu \mathrm{m}$ were formed. Details on the growth, fabrication, and low temperature characterization are reported elsewhere. ${ }^{14,15}$

The Hall sensors were characterized by measuring the longitudinal $\left(V_{x x}\right)$ and transverse $\left(V_{x y}=V_{H}\right)$ voltages at room temperature in dark environment. The transverse (Hall) voltage, $V_{H}$, was measured in fields up to $0.5 \mathrm{~T}$ [Fig. 1(a)]. The sensitivity (Hall coefficient, $R_{H}$ ) [Fig. 2(a)] of the device can be determined by dividing the gradient of the slope ( $V_{H}$ vs B) by $I_{\text {bias. }}$.

For noise measurements, $V_{x y}$ leads were directly connected to the input of a fast Fourier transform spectrum analyzer in a differential (A-B) mode. All the devices were measured in the range $1-4600 \mathrm{~Hz}$ [Fig. 1(b)]. The smaller devices $(\leq 1 \mu \mathrm{m})$ exhibit a more pronounced $1 / f$ (flicker) noise in comparison to larger devices. This is mostly related to the significantly higher resistance of smaller devices, as resistance fluctuations induce a greater effect for devices with higher resistance, hence, larger flicker noise. For example, a typical 500-nm device has significantly larger $R_{4}$, as compared to 5 and $10 \mu \mathrm{m}$ devices (Table I). In comparison, the $R_{4}$ for InSb devices of a comparable size is considerably lower than that of a typical $500-\mathrm{nm}$ graphene device (Table II). On the other hand, recent publication by Tang et al. ${ }^{13}$ showed that $5-\mu \mathrm{m}$ devices made of chemical vapor deposited (CVD) graphene on $\mathrm{SiO}_{2} / n$-Si substrate has twice larger two-terminal resistance $R_{2} \sim 100 \mathrm{k} \Omega$, (Table III) as compared to our 5- $\mu \mathrm{m}$ epitaxial graphene device $\left(R_{2} \sim 50 \mathrm{k} \Omega\right)$. The inset in Fig. 1(b) shows the $1 /$ cross size dependence of the white noise level at zero bias current. It is governed by the Johnson-Nyquist (thermal) noise and, therefore, related to resistance of the device. Noise drops off with the cross width, but saturates at $S_{n} \sim 20 \mathrm{nV} /$ $\sqrt{ } \mathrm{Hz}$ for crosses larger than $5 \mu \mathrm{m}$ wide. When a bias current is applied across the device, the $1 / f$ noise becomes the main contribution to the overall noise, providing a greater effect on smaller devices with significantly higher resistances. For a typical 500$\mathrm{nm}$ wide graphene device the white noise is comparable to our 600-nm wide state-of-the-art InSb device (Tables I and II).

The minimum detectable field, $B_{\mathrm{min}}$, of the sensors was determined by using

$$
B_{\min }=\frac{S_{n}}{R_{H} I_{\text {bias }}},
$$

${ }^{a)}$ Electronic mail: olga.kazakova@npl.co.uk. 

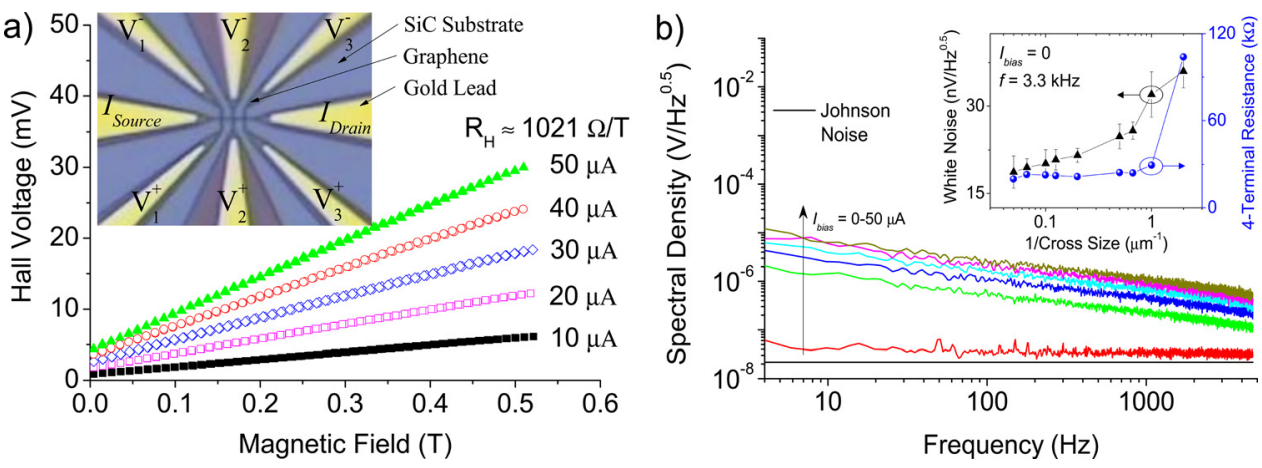

FIG. 1. (Color online) (a) Room temperature field dependence of the DC Hall voltage, $V_{H}$, for a 1- $\mu \mathrm{m}$ cross. Inset shows the optical image for a $0.5-\mu \mathrm{m}$ wide graphene device. $V_{1}, V_{2}$, and $V_{3}$ correspond to crosses 1, 2, and 3, respectively. (b) Room temperature voltage noise spectrum for a 1- $\mu \mathrm{m}$ cross, $I_{\text {bias }}=0-50 \mu \mathrm{A}$. The black line represents the Johnson-Nyquist noise limit. Inset shows the $1 /$ cross size dependence of the white voltage noise, which was averaged over three crosses of the same size at $f=3.3 \mathrm{kHz}$ and $I_{\text {bias }}=0$, and the 4-terminal resistance. The lines are guides for the eye.

a)

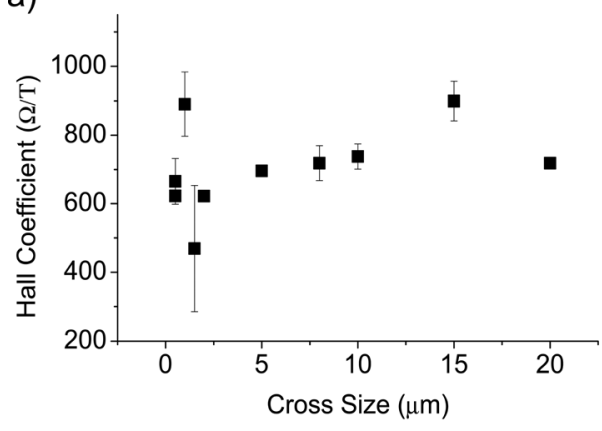

b)

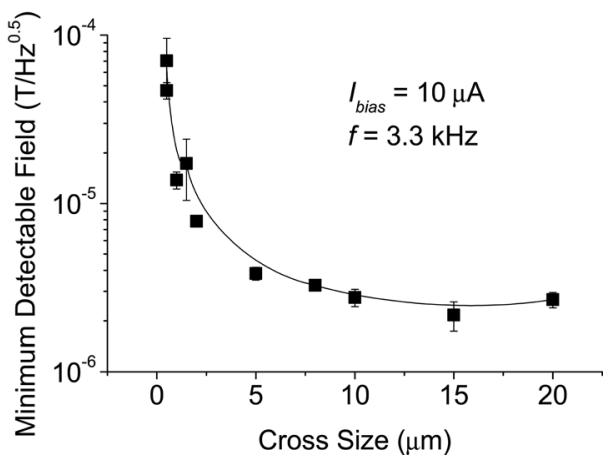

FIG. 2. (a) Size dependence of the Hall coefficient, $R_{H}$, which is averaged for three crosses of the same size. The $R_{H}$ was determined by ramping the magnetic field up to $0.5 \mathrm{~T}$ for $I_{\text {bias }}=10-50 \mu \mathrm{A}$. The $V_{H}$ measurements were performed at five different bias currents for consistency and averaged together. (b) Minimum detectable field vs cross size at $f=3.3 \mathrm{kHz}$ and $I_{\text {bias }}=10 \mu \mathrm{A}$.
TABLE I. Summary of data for a single cross of $0.5,5.0$, and $10.0 \mu \mathrm{m}$ epitaxial graphene devices.

\begin{tabular}{lrcccccr}
\hline \hline $\begin{array}{l}\text { Size } \\
(\mu \mathrm{m})\end{array}$ & $\begin{array}{c}R_{4} \\
(\mathrm{k} \Omega)\end{array}$ & $\begin{array}{c}R_{H} \\
(\Omega / \mathrm{T})\end{array}$ & $\begin{array}{c}N \\
\left(\mathrm{~cm}^{-2}\right)\end{array}$ & $\begin{array}{c}\mu_{e} \\
\left(\mathrm{~cm}^{2} / \mathrm{Vs}\right)\end{array}$ & $\begin{array}{c}S_{n} \\
\left(I_{\mathrm{bias}}=0 \mu \mathrm{A}\right) \\
(\mathrm{nV} / \sqrt{\mathrm{Hz}})\end{array}$ & $\begin{array}{c}B_{\min } \\
\left(I_{\mathrm{bias}}=10 \mu \mathrm{A}\right) \\
(\mu \mathrm{T} / \sqrt{\mathrm{Hz}})\end{array}$ & $\begin{array}{c}\lambda \\
(\mathrm{nm})\end{array}$ \\
\hline 0.5 & 104 & 640 & $9.7 \times 10^{11}$ & 617 & 32.0 & 49.3 & 6.9 \\
5.0 & 22 & 711 & $8.8 \times 10^{11}$ & 2643 & 19.9 & 3.9 & 28.5 \\
10.0 & 23 & 790 & $7.9 \times 10^{11}$ & 2789 & 18.9 & 2.5 & 27.6 \\
\hline \hline
\end{tabular}

TABLE II. Summary of data for $0.6-\mu \mathrm{m}^{12,16,17}$ and $5.0-\mu \mathrm{m}$ InSb devices.

\begin{tabular}{lrcccccc}
\hline \hline $\begin{array}{l}\text { Size } \\
(\mu \mathrm{m})\end{array}$ & $\begin{array}{c}R_{4} \\
(\mathrm{k} \Omega)\end{array}$ & $\begin{array}{c}R_{H} \\
(\Omega / \mathrm{T})\end{array}$ & $\begin{array}{c}N \\
\left(\mathrm{~cm}^{-2}\right)\end{array}$ & $\begin{array}{c}\mu_{e} \\
\left(\mathrm{~cm}^{2} / \mathrm{Vs}\right)\end{array}$ & $\begin{array}{c}S_{n} \\
\left(I_{\text {bias }}=0 \mu \mathrm{A}\right) \\
(\mathrm{nV} / \sqrt{\mathrm{Hz}})\end{array}$ & $\begin{array}{c}B_{\min } \\
(\mu \mathrm{T} / \sqrt{\mathrm{Hz}})\end{array}$ & $\begin{array}{c}\lambda \\
(\mathrm{nm})\end{array}$ \\
\hline 0.6 & 9 & 1106 & $1.2 \times 10^{11}$ & 13000 & 28.0 & $5.4\left(I_{\text {bias }}=5 \mu \mathrm{A}\right)$ & 52.5 \\
5.0 & 12 & 974 & $8.7 \times 10^{10}$ & 8322 & 55.3 & $6.5\left(I_{\text {bias }}=10 \mu \mathrm{A}\right)$ & 27.4 \\
\hline \hline
\end{tabular}

where $S_{n}$ is the noise power spectral density, $R_{H}$ is the Hall coefficient and $I_{\text {bias }}$ is the bias current. For $5-\mu \mathrm{m}$ epitaxial graphene devices, $B_{\min } \approx 4 \mu \mathrm{T} / \sqrt{ } \mathrm{Hz}$ at $f=3.3 \mathrm{kHz}$ and $I_{\text {bias }}=10 \mu \mathrm{A}$, which is comparable with our previous results on InSb devices ${ }^{12}$ (measured under similar conditions) and 10 times better than CVD graphene device (Table III). ${ }^{13}$ However, for smaller epitaxial graphene devices (500-nm), sensitivity drops to $B_{\min } \approx 50 \mu \mathrm{T} / \sqrt{ } \mathrm{Hz}$ at $f=3.3 \mathrm{kHz}$ and $I_{\text {bias }}=10 \mu \mathrm{A}$, i.e., worse than InSb devices of the similar size due to the high resistance, which increases the flicker noise. This fact implies necessity of improvements in the fabrication process of small epitaxial graphene devices. Figure 2(b) shows that the minimum detectable field for smaller graphene devices decreases exponentially with increasing cross width (up to $5 \mu \mathrm{m}$ ) and saturates for $10 \mu \mathrm{m}$, at which point the sensitivity is determined by the thermal noise of the device.

The carrier density, $n$, was defined as $n=1 / e R_{H}$, where $e$ is electron charge, and the carrier mobility was defined as

$$
\mu_{e}=\frac{I_{\text {bias }} R_{H}}{V_{x x}} \times \text { no. of squares. }
$$




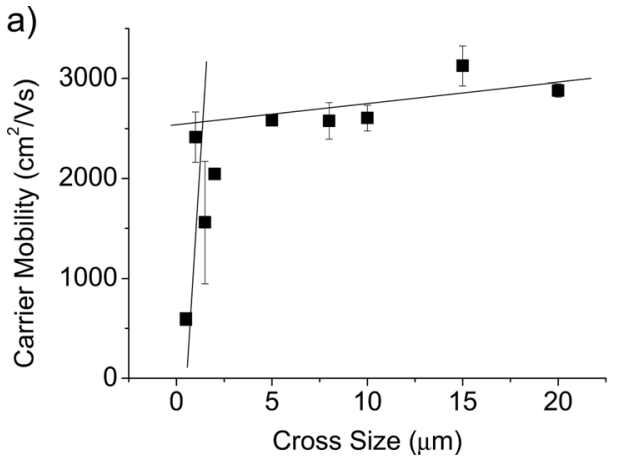

The number of squares is related to the resistivity in the following way $R=(\rho / t)(L / W)$, where the resistivity, $\rho$, is divided by the sheet thickness, $t$, to give sheet resistance, $R_{s}$, and length, $L$, divided by width, $W$, to give number of squares, hence $R=R_{s} \times$ no of squares. The carrier mobility relation, $\mu_{e}=(t / \rho) R_{H}$, then becomes

$$
\mu_{e}=\frac{R_{H}}{R_{s}}=\frac{R_{H}}{R} \times \text { no. of squares, }
$$

where $R=R_{4}$. Figure 3(a) shows that $\mu_{e} \approx 3000 \mathrm{~cm}^{2} / \mathrm{Vs}$ for devices that are $\geq 1 \mu \mathrm{m}$, whereas for 500 -nm devices $\mu_{e}$ sharply drops to $\approx 600 \mathrm{~cm}^{2} /$ Vs. Even the largest values are significantly smaller than those in InSb samples (Tables I and II). Dependence of $1 / n$ on $\mu_{e}$ [Fig. 3(b)] also demonstrates two distinct mechanisms that determine the mobility. This implies that for smaller devices the dominant mechanism is primarily dependent on the channel width, whereas for larger devices $(\geq 1 \mu \mathrm{m})$ it is predominantly material dependent. The calculated carrier mean free path, ${ }^{18}$

$$
\lambda=\frac{h}{2 e} \mu_{e}\left(\frac{n}{\pi}\right)^{1 / 2}
$$

where $h$ is Plank's constant, gives $\lambda \approx 7 \mathrm{~nm}$ for a typical 500-nm graphene device and $\lambda \approx 20-30 \mathrm{~nm}$ for the other devices. This shows that at room temperature the electron transport is strongly diffusive for all the devices. The mobility is also size-dependent, signifying importance of both intrinsic (i.e., defects in crystalline structure of graphene and SiC substrate, impurities, grain boundaries, etc.) and extrinsic (i.e., unoptimized fabrication) factors in the device performance. Nonuniformity of mono- or bilayer graphene across the substrate $^{19}$ and interactions between graphene and $\mathrm{SiC}$ substrate can all lead to significant increase in scattering. The carrier density is also affected as seen in Fig. 2(a), For comparison, in graphene obtained by mechanical cleavage on top of silicon dioxide substrate with $\mu_{e} \approx 10000 \mathrm{~cm}^{2} / \mathrm{Vs}$ and $n$ $\approx 10^{12} \mathrm{~cm}^{-2}$ (Ref. 20), the carrier mean free path can be as large as $\sim 100 \mathrm{~nm}$ at room temperature and $\lambda \approx 1.2 \mu \mathrm{m}$ at $\sim 5 \mathrm{~K}^{18}$

TABLE III. Summary of data for 5- $\mu$ m CVD graphene device. ${ }^{13}$

\begin{tabular}{llcc}
\hline \hline $\begin{array}{l}\text { Size } \\
(\mu \mathrm{m})\end{array}$ & $\begin{array}{l}R_{2} \\
(\mathrm{k} \Omega)\end{array}$ & $\begin{array}{l}R_{H} \\
(\Omega / \mathrm{T})\end{array}$ & $\begin{array}{c}B_{\min }\left(I_{\text {bias }}=3 \mu \mathrm{A}\right) \\
(\mu \mathrm{T} / \sqrt{ } \mathrm{Hz})\end{array}$ \\
\hline 5.0 & 100 & 310 & 43.0 \\
\hline \hline
\end{tabular}

FIG. 3. (Color online) (a) Size dependence of the carrier mobility averaged for three crosses of the same size at room temperature. Lines are guides for the eye only. (b) Dependence of the inverse carrier density, $1 / n$, on the carrier mobility. The group of data points in red (smaller) circle are measurement for $0.5-\mu \mathrm{m}$ devices and the points in black (larger) circle are for 1 to $20 \mu \mathrm{m}$ devices. Each common colored set of data points represents crosses 1, 2, and 3 for an individual device.

Thus, we demonstrated epitaxial graphene magnetometers with room-temperature Hall coefficient approaching that of state-of-the-art semiconductor devices of a similar carrier density and size. We have also demonstrated that epitaxial graphene devices offer lower resistivity and an order of magnitude better sensitivity to magnetic field when compared to CVD graphene Hall sensors. Large epitaxial graphene sensors ( $\geq 5 \mu \mathrm{m}$ ) are more sensitive to magnetic field than smaller sensors due to inherently higher resistances of the latter. While epitaxial graphene devices still provide significantly lower mobility and mean free path than exfoliated graphene devices, their macroscopic arrays can be straightforwardly prepared over large areas, making epitaxial graphene readily compatible with $\mathrm{Si}$ electronic processing. Readily available epitaxial graphene devices can be very attractive for chemical and biosensing as well as for magnetic storage applications.

\section{ACKNOWLEDGMENTS}

This work has been developed under the EU FP7 Project JRP IND 11 Metrology for Advanced Industrial Magnetics (MetMags).

${ }^{1}$ E. H. Hall, Am. J. Math. 2, 287 (1979).

${ }^{2}$ G. Masson, D. Frachon, T. Dorge, Y. Ronnat, and R. Arlot, Sens. Lett. 7, 451 (2009).

${ }^{3}$ Z. Bing, X. Du, and J. Sun, IEEE Trans. Power Electron. 26, 1800 (2011).

${ }^{4}$ A. Simpkins and E. Todorov, Proceedings of the American Control Conference, 1948-1955 (2010).

${ }^{5}$ J. Heremans, J. Phys. D: Appl. Phys. 26, 1149 (1993).

${ }^{6}$ A. Sandhu, H. Masuda, A. Oral, and S. J. Bending, Jpn. J. Appl. Phys. 40, 4321 (2001)

${ }^{7}$ G. Boero, M. Demierre, P. Besse, and R. Popovic, Sens. Actuators, A 106, 314 (2003).

${ }^{8}$ L. Ejsing, M. F. Hansen, A. K. Menon, H. A. Ferreira, D. L. Graham, and P. P. Freitas, Appl. Phys. Lett. 84, 4723 (2004).

${ }^{9}$ K. Togawa, IEEE Trans. Magn. 41, 3661 (2005).

${ }^{10} \mathrm{P}$. Manandhar et al., Nanotechnology 20, 355501 (2009).

${ }^{11}$ A. Sandhu, Adarsh, H. Handa, and M. Abe, Nanotechnology 21, 442001 (2010).

${ }^{12}$ O. Kazakova, V. Panchal, J. Gallop, P. See, D. C. Cox, M. Spasova, and L. F. Cohen, J. Appl. Phys. 107, 09E708 (2010).

${ }^{13}$ C.-C. Tang, M.-Y. Li, L. J. Li, C. C. Chi, and J. C. Chen, Appl. Phys. Lett. 99, 112107-1 (2011).

${ }^{14}$ A. Tzalenchuk et al., Nature Nanotechnol. 5, 186-9 (2010).

${ }^{15}$ A. Tzalenchuk et al., Solid State Commun. 151, 1094 (2011).

${ }^{16}$ L. Di Michele, C. Shelly, J. Gallop, and O. Kazakova, J. Appl. Phys. 108, 103918 (2010).

${ }^{17}$ L. Di Michele, C. Shelly, P. de Marco, P. See, D. Cox, and O. Kazakova, J. Appl. Phys. 110, 063916 (2011).

${ }^{18}$ K. Bolotin, K. Sikes, Z. Jiang, M. Klima, G. Fudenberg, J. Hone, P. Kim, and H. Stormer, Solid State Commun. 146, 351 (2008).

${ }^{19}$ T. Burnett, R. Yakimova, and O. Kazakova, Nano Lett. 11, 2324 (2011).

${ }^{20}$ A. K. Geim and K. S. Novoselov, Nature Mater. 6, 183 (2007). 